\title{
A REVIEW ON THIRD EYE
}

\section{RAJESH KUMAR, ASHOK KUMAR SAHOO \& SAGAR SAHOO}

Institute of Technical Education and Research, Siksha 'O'Anusandhan Deemed to be University, Bhubaneswar, Odisha, India

In this paper, we discuss the third eye. In this propelled world, where individuals begins opening new ways to take care of their issues they confronting, to satisfy their prerequisites and to make their creative ability to the real world. In this procedure, a large number of us begins looking towards the sky, beginning from Galileo to the 100 satellites dispatch by ISRO (Indian Space Research Organization) in one go. People have accomplished so much and gained such a great amount from the nature itself, by looking to the working of the nature and uncovering its privileged insights. We have figured out how the universe is begun from a solitary and boundlessly little point in space till it's very development in which we are living now and making the inquiry, 'Where do this chaos originates from?'Huge numbers of the extraordinary researchers ask a similar inquiry, and this very erg to discover the appropriate response took them to the introduction of innovation which they use to find the solution of their inquiries. Numerous speculations were made and in the end vanish when man began sending its space creates in space for more profound and further developed learning and comprehension of the universe. Through this very paper, we may investigate the space from an alternate perspective, or we can say from all perspectives which are required by our researcher for better comprehension of the working of the universe.

KEYWORDS: Technology \& Future
\end{abstract}

Received: Apr 03, 2019; Accepted: Apr 23, 2019; Published: Jun 08, 2019; Paper Id.: IJMPERDJUN2019155

\section{INTRODUCTION}

Space science, astronomy, space inquire about, space perception, profound space, space create configuration, RTG's, 3-D imaging, moveable cameras, attractive field, moveable rockets, round structure, 8 constant pictures, temperature controlled, 360 degree see, light weight, long lifetime, self-kept up.

\section{THE DETAILED DESCRIPTION}

Nature, before getting specifically to the plan of our space creates, we should know about every angle on which our structure will take a shot at. Essentially, in the event that we are structuring our space create for a space program we should know about the circumstances which the space specialty will confront[1].

As the space is a virus place, here we are close to our well-known star (The Sun), which keep us alive and now we have figured out how to utilize its vitality for our very own advantages. Truly, the sun powered cells. 'Photons' which drives the satellites, give them power fundamentally the sun is keeping us alive, yet imagine a scenario in which, our space make needs to go into the profound space. Our capacity supplier won't be there to give us the endowment of light and vitality to keep our ventures moving [2].

So, when we move far from the earth our first check is coming specifically from the sun itself, 'the Solar flares', which is consistently transmitted by the sun can be another string to the space create on the grounds that the 
satellite which are being used, are for the most part moving around the Earth and we realize that the Earth has its very own attractive field, which shields all of us from the sun powered breezes, the charged particles moving at the speed of light and can cause a profound and regularly enduring impact on any planet. Model: - our extremely next neighbor 'The Mars' we can see the strong evidence of presence of water on that planet yet been a littler planet when contrasted with the Earth it cools quicker, its center quits moving bringing about the loss of the planets attractive field. When the attractive shield vanishes, the sun based breezes makes the water to bubble lastly dissipates into the space leaving the planet a dead bundle of residue[3]. The equivalent can be the situation with our space make. The sun oriented breezes can make its circuit dead forever.

"Which influence us to comprehend the significance of attractive field lines to ensure our space make"?

Another and an intriguing certainty, which I consider to be essential is the job of 'ozone' (later it will demonstrate valuable in the plan of the space make) in our environment. The ozone particle in the air essentially comprises of three oxygen iotas bound together and having the synthetic equation O3. Anyway, it has an extremely intriguing job which is gainful. It assimilates the greater part of the ultra-violet radiations (UV-B). This retention of bright radiations by $\mathrm{O} 3$ atom makes a wellspring of warmth, which really shapes the stratosphere itself, the locale in which the temperature ascends as one goes to higher elevation. Ozone subsequently assumes an essential job in the temperature upkeep of the planet's climate[4].

"In this manner, a reasonable measure of ozone gas will assist us with maintaining the temperature of the space make, when the power unit isn't being used".

As this paper is composed for the basic plan, which will give a superior comprehension of the nature and the articles which are moving as per the laws of the nature. For the investigation of the universe, we should have investigated and for going all the more closer to the divine bodies implies we need to move far from our mom planet, the profound space which isn't as protected as it imagines it to be. No sunlight based light, no temperature support, no attractive field are the serious issues which our space create face, while going toward the external close planetary system [5].

Here, the utilization of atomic vitality is an unquestionable requirement and before utilizing it we should comprehend its significance in detail.

Atomic vitality tackles the vitality from the rot of radioactive materials, normally 'Uranium-235'. Atomic vitality is the vitality delivered by the mass to vitality transformation. Energy=mass*(speed of light, squared) this is the place the shared characteristic finishes however the utilization of atomic vitality in space, normally either little splitting framework or radioactive rot for warmth or power is normal. One normal sort of radioisotope thermoelectric generator 'Mossbauer spectrometer', which has been utilized on many space tests and on, kept an eye on lunar mission. Another is little parting reactor for satellites which are near the Earth is 'TOPAZ atomic reactor' which gives the warmth from the radioactive rot of material for a considerable length of time. Russia sends in excess of 40 reactors into the space by utilizing 'TOPAZ-II reactor' having an intensity of in excess of 10 kilowatts. The 'Romashka reactor' utilizes Uranium as its fuel and creates power specifically. NASA tried it for over 42 days in its labs. For the development of the tests and rockets Nuclear power is the best decision as atomic electric rockets, radioisotope electric impetus are been utilized by NERVA program[6].

Presently, we as a whole are having the regular inquiry that why not like to utilize sun oriented power as it's the least expensive, however atomic power offers incredible points of interest in numerous regions, not at all like sun oriented 
cells. Nuclear power framework work freely of the daylight which is vital for the profound space investigation. One more advantage that makes the atomic ideal in the space is its weight. It is light load when contrasted with the sun oriented cells. Subsequently, atomic power framework takes up significantly less space than sun oriented power framework. Conservative space create are simpler to situate and coordinate in space, so atomic vitality can control both life backing and impetus framework, so this framework can viably diminish cost and time both[7].

The sort of framework that will be gainful to help our rocket, and been utilized in numerous projects is 'Radioisotope System'. For over fifty years, "Radioisotope thermoelectric generators" (RTG's) have been utilized by man as a power source in space. RTG's offers numerous advantages some are: self - kept up, generally protected and is solid under cruel conditions, and can work effectively for quite a long time. Many RTG's are been working for over 20 years in space[8].

For our space craft's power source, I have some choices: -

- $\quad$ SNAP-19

- $\quad$ SNAP-27

- MHW-RTF

- $\quad$ GPHS-RTG

- MMRTG

So, the nuclear generators are developing as a great speed. We can have: -

Efficiency: - 20-26\%.

Power: - 130 Watts (from near future nuclear generator 'ASRG' Advanced Sterling Radioisotope Generator).

Total Mass: - 70 pounds (32 kilograms).

Fuel: - 2.7 pounds (1.2 kilograms) of plutonium dioxide protected in two General Purpose Heat Source Modules.

Dimensions: - 2.5 feet long (76 centimeters; 46 by 39 centimeters).

\section{Design Lifetime: - At least 17 years.}

(Information from NASA's use of radioisotope power system for space exploration)

(For better understanding, we suggest the reader to have a constant look at the design attached along.)

Now, when we are equipped with the knowledge to power our space craft, now we will use this power in five major domains:

- $\quad$ To move our space craft.

- For the movement of rockets.

- $\quad$ To power the control unit.

- To power the magnetic unit.

- To power the cameras. 


\section{To Move the Space Craft}

To move or to drive the item forward in a specific heading is known as impetus and any framework utilizes rocket impetus technique for its development. Any framework and motors in space carries on as indicated by Sir Isaac Newton's third law of movement: "Each activity delivers an equivalent and inverse response". At the point when a rocket shoots fuel out toward one side, this drives the rocket forward, which implies no air (no medium) is required for its development[9].

So, space create impetus is any strategy used to quicken space make and fake satellite, so it is a functioning territory of research. There are a wide range of strategies, and every strategy has its very own advantages and points of interest. Aside from specifically utilizing fuel for drive of the rocket we may utilize each other technique for speeding up of the space make since when any space create is send to the space, it needs to move and set on the right way to achieve its goal. Most shuttle do this by compelling a gas from the backside of the rocket at extremely rapid. This looked for of motor is known as rocket motor [10].

As we are examining the utilization of atomic power source, we will utilize atomic fueled rockets so as to move our space make on wanted way. We realize that the atomic controlled rockets will give push to the space create, yet it will utilize a heap of fuel i. e., the space specialty will move at a specific speed just, which would not be adequate to set it in profound space however we have an answer for this issue. To set aside extra cash, fuel and time when shooting so far-away planets, for example, Jupiter, Saturn, Neptune, and so forth (generally external nearby planetary group). The space makes whip around a planet (state Venus) and utilize its gravity to get a speed support. This abbreviates the time, set aside some cash spare the fuel and keeping in mind that working with an atomic power source, we generally have a restricted timeframe and the work must be done inside the constrained timeframe[11].

\section{For the Movement of Rockets}

For the rakish movement of the space create, we need to give the drive to the space make by shooting the fuel at a point. To give this impetus to the shuttle, we need to set the rockets at a point. So, for the development of the rockets, we need vitality from the atomic power source, we utilize its vitality to driving the six rockets we have toward the finish of every leg. I clarify all the unpredictable movement with the assistance of graphs (appended along).

\section{To Power the Control Unit}

For all the correspondence between the space make and the general population controlling it, there is a unit at the focal point of the space create which is the controlling and working arrangement of the space make. This unit will give guidance, which we customized to the diverse parts of the space art to play out their separate capacities in the trained way. For driving this unit, we utilize our atomic power source, which is equipped for changing over the atomic vitality to the electrical vitality. For playing out the undertakings and to watch the external immaculate universe is in an unexpected way.

\section{To Power the Magnetic Unit}

As we have talked about it before, the significance of attractive field lines for any planet and for any space make working up in the space and to secure out space create, I present the attractive unit in our space make (the chart appended along). the sufficient electro-magnets are fit for delivering the attractive field lines. This will give the attractive field sufficiently able to shield the cameras from the sun oriented flares and the electrical circuits' structure, the charged particles and the infinite beams from the outer universe and the sun itself. 


\section{To Power the Cameras}

The most dominant and most vital piece of this plan is the cameras of the space create, through which all the data from the outer universe will be passed on to us. The eight ground-breaking cameras present at the sides of the focal unit. These cameras can be utilized in various modes as wanted by the client to get pictures of the item in the noticeable light locale just as in alternate areas of the light on the grounds that, in the present-day situation for gathering the pictures in various spaces of light, we need diverse telescopes, with the assistance of such an extraordinary machine if an imperative change in a gas planet like Jupiter or Saturn for its profound examination, we need pictures in its distinctive spaces. The pictures of higher quality and having the data are never assembled. Also, to get the ideal perspective of the items which the space specialty will concentrate on, we make the cameras substantially more moveable and can be controlled through the controlling unit. As we can assemble substantially nittier gritty data by survey, the article in 3-dimentional see, we can join the two pictures from the 8-cameras and can have the ideal 3-dimentional pictures of the item. We can take the pictures of the midget planets, the space rocks, the comets, the little satellites and substantially more in the outside universe [12].

\section{Importance of Ozone in the Space Craft}

As we have talked about before, the ozone has the property to trap heat. Along these lines, a reasonable measure of ozone in the space art will be useful in the temperature control on the grounds that the profound space is a lot colder than the stops shafts on the earth. Along these lines, an appropriate measure of ozone will keep the temperature up when the driving unit isn't being used.

This gas ought to be expelled just before beginning the RTG (the control unit), as it can make the temperature to be a lot higher and may burst into flames. Hence, just before beginning up the power unit, this gas ought to be spilled in the outside condition for the wellbeing of our task. So, a brilliant utilization of ozone will be useful in the space.

\section{CONCLUSIONS}

Through this special structure, we can have a conceivably exceptionally solid plan, which will demonstrate helpful in the examination. It's one of a kind legs having 6 rockets will permit this space art to move any ideal way without pondering the introduction of the space make. It will act naturally kept up and safe. No compelling reason are to keep up the space create. It will work for a considerable length of time with no damage to it. It can took pictures of the planets of the gas goliaths at same time in unmistakable range along in alternate areas of light to comprehend the responses going underneath the gassy air in the monsters. We can get the diverse pictures of the moons of the gas mammoths, which can be a spot to live, as a strong confirmation of warm seas underneath its surface. An extremely far planet can be profoundly comprehended with this space create in light of the fact that the pictures, which we have about this planet are not giving enough data about this little planet. A proof of water is there on Pluto as well. This space make is protected from the sunlight based flares as its attractive unit will give wellbeing to this space create. With this plan, we can turn this space make at practically any pivot, which I believe is never be conceivable with any space make in mankind's history. Since we are utilizing the atomic vitality, the space art will be anything but difficult to orientate about, and will be exceptionally light weight which will be extremely gainful of any space create. This plan will give a lot of immaculate data about this very. 


\section{REFERENCES}

1. C. Wolfram, E. Stahlberg, and N. Pfeiffer, "Patient-Reported Nonadherence with Glaucoma Therapy,” J. Ocul. Pharmacol. Ther.

2. S. R. Benoit, B. Swenor, L. S. Geiss, E. W. Gregg, and J. B. Saaddine, "Eye Care Utilization Among Insured People With Diabetes in the US, 2010-2014,” Diabetes Care, vol. 42, no. 3, pp. 427-433, Mar. 2019.

3. E. Bozkurt, B. Cakir, E. Celik, E. Dogan, T. Ucak, and G. Alagoz, "Correlation of the aqueous humor total antioxidant capacity, total oxidant status, and levels of IL-6 and VEGF with diabetic retinopathy status," Arq. Bras. Oftalmol., vol. 82, no. 2, pp. 136-140, 2019.

4. N. Bravo-Gil, I. Marcos, A. Gonzalez-Meneses, G. Antinolo, and S. Borrego, "Expanding the clinical and mutational spectrum of germline ABL1 mutations-associated syndrome: A case report," Medicine (Baltimore)., vol. 98, no. 10, Mar. 2019.

5. J. R. Goldschmied, P. Cheng, R. Hoffmann, E. M. Boland, P. J. Deldin, and R. Armitage, "Effects of slow-wave activity on mood disturbance in major depressive disorder," Psychol. Med., vol. 49, no. 4, pp. 639-645, Mar. 2019.

6. Sharma, A., \& Thakur, D. (2017). A review on WLANs with radio-over-fiber technology. International Journal of Electronics and Communication Engineering (IJECE), 6(5), 1-6.

7. Izharuddin, “Does sociology need decolonizing?,” Int. Sociol., vol. 34, no. 2, pp. 130-137, Mar. 2019.

8. Kaesmann-Kellner, K. Moslemani, and B. Seitz, "Clinical and Genetic Characteristics of Ocular Developmental Disorders: MAC-Spectrum, Anterior Segment Dysgenesis,” Klin. Monbl. Augenheilkd., vol. 236, no. 3, pp. 269-285, Mar. 2019.

9. N. G. Kavallieratos, C. G. Athanassiou, M. C. Boukouvala, and G. T. Tsekos, "Influence of different non-grain commodities on the population growth of Trogoderma granarium Everts (Coleoptera: Dermestidae)," J. Stored Prod. Res., vol. 81, pp. 31-39, Mar. 2019.

10. Koornneef, A. Kraal, and M. Danel, "Beginning readers might benefit from digital texts presented in a sentence-by-sentence fashion. But why?," Comput. Human Behav., vol. 92, pp. 328-343, Mar. 2019.

11. Berliandaldo, M., Hidayat, M., \& Hidayat, A. (2017). Gap Analysis of Knowledge Management of Cibinong Science \& Technology Park-Indonesia. International Journal of Business and General Management (IJBGM), 6(6), 37-46.

12. J. Lim et al., "Intraocular Pressure Measurement by Rebound Tonometry (TonoVet) in Normal Pigeons (Columba livia)," J. Avian Med. Surg., vol. 33, no. 1, pp. 46-52, Mar. 2019.

13. D. J. Mathew, A. Anuradha, S. A. W. Low, A. Belkin, Y. M. Buys, and G. E. Trope, "Long-term Follow-up of Ahmed Glaucoma Valve Tube Position Changes,” J. Glaucoma, vol. 28, no. 3, pp. 276-280, Mar. 2019.

14. W. Moehring et al., "The association between sleep and dual-task performance in preterm and full-term children: an exploratory study," SLEEP Med., vol. 55, pp. 100-108, Mar. 2019. 